\title{
Modelling Cell Migration and Adhesion During Development
}

\author{
Robin N. Thompson • Christian A. Yates • \\ Ruth E. Baker
}

Received: 14 May 2012 / Accepted: 27 September 2012 / Published online: 19 October 2012

(C) Society for Mathematical Biology 2012

\begin{abstract}
Cell-cell adhesion is essential for biological development: cells migrate to their target sites, where cell-cell adhesion enables them to aggregate and form tissues. Here, we extend analysis of the model of cell migration proposed by Anguige and Schmeiser (J. Math. Biol. 58(3):395-427, 2009) that incorporates both cell-cell adhesion and volume filling. The stochastic space-jump model is compared to two deterministic counterparts (a system of stochastic mean equations and a non-linear partial differential equation), and it is shown that the results of the deterministic systems are, in general, qualitatively similar to the mean behaviour of multiple stochastic simulations. However, individual stochastic simulations can give rise to behaviour that varies significantly from that of the mean. In particular, individual simulations might admit cell clustering when the mean behaviour does not. We also investigate the potential of this model to display behaviour predicted by the differential adhesion hypothesis by incorporating a second cell species, and present a novel approach for implementing models of cell migration on a growing domain.
\end{abstract}

Keywords Mathematical modelling · Cell-cell adhesion · Differential adhesion · Cell sorting · Domain growth

R.N. Thompson $(\bowtie) \cdot$ C.A. Yates $\cdot$ R.E. Baker

Centre for Mathematical Biology, Mathematical Institute, University of Oxford, 24-29 St Giles',

Oxford OX1 3LB, UK

e-mail: rnt22@cam.ac.uk

Present address:

R.N. Thompson

Theoretical and Computational Epidemiology Group, Department of Plant Sciences, University of

Cambridge, Downing Street, Cambridge CB2 3EA, UK 


\section{Introduction}

Cell migration and adhesion are vital during biological development: cells migrate to their target sites, and cell-cell adhesion enables them to aggregate and form cohesive tissues. Further, cell distributions can be controlled by differing expression levels of cell adhesion molecules (Alberts et al. 1994; Foty and Steinberg 2005).

Models of cell migration generally come in two complementary forms: stochastic, individual-based or deterministic, population-based (Erban and Othmer 2004). Simulations of stochastic models often require more computational work, but do include the randomness that is often prevalent in biological systems. Population-based models, which usually involve systems of partial differential equations (PDEs), generally not only require less computational work to obtain a numerical solution, but one can also use many tools from PDE analysis to explore their behaviour. Further, population-level readouts are sometimes more useful: for example, a surgeon is interested in the population-level behaviour of a tumour (particularly, how fast it will grow), rather than the movements of individual cells. Whilst models of cellcell adhesion considered previously have predominantly been discrete, individualbased (Simpson et al. 2010b; Khain et al. 2011), there are relatively few continuum, population-based models (Armstrong et al. 2006; Anguige and Schmeiser 2009). Linking both discrete and continuous models of cell-cell adhesion, as we do here, is even less common (Simpson et al. 2010a).

There are several types of individual-based model. One of the most commonly used is the space-jump model, where each cell moves around in space on a lattice, jumping from its current compartment to a neighbouring one (Baker et al. 2010; Berg 1993; Othmer and Stevens 1997; Othmer et al. 1988). Other models include velocity-jump models (Berg 1975; Othmer et al. 1988), where each cell repeatedly jumps between different velocities. Both of these types of individual-based model have been connected to population-based models (Erban and Othmer 2004; Othmer and Hillen 2011; Othmer and Stevens 1997).

The Keller-Segel model (Keller and Segel 1971a; Patlak 1953; Murray 2002) is a commonly used population-based model for cell migration and adhesion due to chemotaxis. Various biological phenomena, including the movement of Escherichia coli (Keller and Segel 1971b; Brenner et al. 1998) and Dictyostelium discoideum (Keller and Segel 1970; Othmer and Schaap 1998), have been represented using the Keller-Segel model, despite the fact that at the mesoscopic cell level these systems behave in different ways. This further motivates the need for individual-based models: to incorporate biologically accurate descriptions of individual cell motion.

In this paper, we consider a stochastic model for cell migration on a fixed, onedimensional lattice that incorporates volume filling as well as cell-cell adhesion. In Sect. 2.2, the corresponding set of deterministic stochastic mean equations (SMEs) for the average cell density in each compartment of the lattice are considered, as is a deterministic non-linear PDE describing the evolution of cell density across the domain. These systems were initially proposed by Anguige and Schmeiser (2009), and the deterministic versions have been extended to include chemotaxis by Anguige (2011).

We look, in Sect. 3, at cases where the behaviour of the stochastic system is both similar to, and very different from, the behaviour of the deterministic systems. We 
then extend the stochastic model to include two cell species in Sect. 4, and explore whether it can display behaviour predicted by the differential adhesion hypothesis (Foty and Steinberg 2005), which attributes the sorting of different cell species, starting with a random mix of each species, to differing adhesion properties of the various species. Whilst several models have been proposed to describe cell-cell adhesion (such as those by Palsson and Othmer 2000, Simpson et al. 2010b and Armstrong et al. 2006), we show that various cell sorting behaviours can occur in a simple model, via adhesion both between cells of the same species and cells of different species.

In Sect. 5, we implement the stochastic model and its deterministic counterparts on a growing domain, which occurs, for example, during the development of vertebrate embryos (Landman et al. 2003). Whilst there is a range of literature about models of biological systems that include domain growth, particularly continuum models (Crampin et al. 1999; Maini and Solursh 1991; Mooney and Nagorcka 1985), including both cell migration and stochastic domain growth is comparatively rare (Woolley et al. 2011), and has not to our knowledge been considered for models of cell-cell adhesion. We find problems with the previously used compartment-splitting approach to domain growth (Baker et al. 2010) in cases where there is a wide variation in cell density across the domain, and present a novel approach for domain growth in this paper.

\section{Modelling Cell-Cell Adhesion on Fixed Domains}

\subsection{The Stochastic Model}

The discrete model considered will be a mesoscale, individual-based model, that incorporates both cell-cell adhesion and volume filling. Cells move on a onedimensional lattice $x \in[0,1]$ with $k$ lattice points, where each lattice point is assumed to be the midpoint of a compartment in which cells sit. In order to make later implementation of domain growth more straightforward, we define $h_{i}$ as the distance between lattice points $i-1$ and $i$ (for $i$ between 2 and $k$ ), $h_{1}$ as twice the distance between the left-most lattice point and the left edge of our domain, and $h_{k+1}$ as twice the distance between lattice point $k$ and the right edge of the domain.

Initially, we assume that lattice points are equally spaced, so that $h_{i}=h=1 / k$, for $i$ between 1 and $k+1$. We also assume no-flux boundary conditions, and no cell proliferation or death, so that the total number of cells is constant (as is the total cell density, summed over all compartments, since lattice points are equally spaced and the domain is fixed).

Let the vector $\mathbf{n}=\left(n_{1}, n_{2}, \ldots, n_{k}\right)$ represent the number of cells in each compartment, and let $T_{i}^{ \pm}(\mathbf{n})$ be the transition rates for a cell moving right ( + ) or left (-) from compartment $i$ to compartment $i \pm 1$ (we assume nearest neighbour transitions only), so that, on average, the net flux of cells from compartment $i$ to $i+1$, say, is

$$
n_{i} T_{i}^{+}-n_{i+1} T_{i+1}^{-} \text {. }
$$

In this model, for $i=2,3, \ldots, k-1$, we take

$$
T_{i}^{+}(\mathbf{n})=\frac{d}{h^{2}}\left(1-\alpha \frac{n_{i-1}}{S}\right)\left(1-\frac{n_{i+1}}{S}\right),
$$




$$
T_{i}^{-}(\mathbf{n})=\frac{d}{h^{2}}\left(1-\frac{n_{i-1}}{S}\right)\left(1-\alpha \frac{n_{i+1}}{S}\right),
$$

with

$$
\begin{aligned}
& T_{1}^{+}(\mathbf{n})=\frac{d}{h^{2}}\left(1-\alpha \frac{n_{1}}{S}\right)\left(1-\frac{n_{2}}{S}\right), \\
& T_{1}^{-}(\mathbf{n})=0, \\
& T_{k}^{+}(\mathbf{n})=0, \\
& T_{k}^{-}(\mathbf{n})=\frac{d}{h^{2}}\left(1-\frac{n_{k-1}}{S}\right)\left(1-\alpha \frac{n_{k}}{S}\right),
\end{aligned}
$$

as proposed by Anguige and Schmeiser (2009), where $\alpha \in[0,1]$ is the constant adhesion coefficient and $d$ is a constant that can be adjusted so that adhesion occurs on biologically relevant timescales.

We propose this model as a possible explanation of cell clustering behaviour seen in experimental systems. The model could represent, for example, a two-dimensional process, where each compartment represents a thin vertical strip (that is one cell width wide). For simplicity, we assume that cells can only move vertically or horizontally (a common assumption in Cartesian lattice-based models). A cell that sits in compartment $i$ exerts a force on cells which are immediately adjacent to it in compartments $i-1$ and $i+1$. Consequently, ignoring any vertical transitions (since this does not change the compartment in which the cell concerned sits), a cell can move horizontally either left or right. The probability of a cell moving to the right from compartment (vertical strip) $i$ to $i+1$ is reduced if there is a cell immediately on the left of the original cell by the adhesive force: this is the case with probability $n_{i-1} / S$ if there are $n_{i-1}$ cells in compartment $i-1$ (since we do not know exactly where in the compartment the cells sit). The cell cannot move to the right if there is a cell already there (this has probability $n_{i+1} / S$ ). This motivates the transition probabilities above. Other, more complex forms of the transition probabilities above are possible in order to explain cell-cell adhesion, but ours are chosen for their simplicity.

In order for direct comparison with the results of Anguige and Schmeiser to be possible, we set $d$ to unity in this work. Since $\alpha \in[0,1]$, the probability of a particle jumping to the right is reduced if there are many particles to the left (and vice versa), thus representing adhesion (with $\alpha$ determining the strength of adhesion). $S \in \mathbb{N}$ is the carrying capacity of a compartment: it limits the number of cells in each compartment at any time, and represents volume filling. We simulate the model using the Gillespie stochastic simulation algorithm (Gillespie 1977).

\subsection{The Deterministic Models}

SMEs describing the mean cell density in each compartment (where $\left\langle\rho_{i}\right\rangle(t)=$ $\left\langle n_{i}\right\rangle(t) / S$ denotes the mean cell density in compartment $i$ at time $t$, and we assume that the carrying capacity of each compartment, $S$, is proportional to its length) can be derived by conditioning on the state of the stochastic system at time $t$ and considering its state at some short time later, $t+\delta t$ (a master equation formulation, as in 
Baker et al. (2010)). In the derivation, we assume that the variance of the density of cells in each compartment (and corresponding covariances between compartments) is negligible, so that, for example, $\left\langle n_{i} n_{j}\right\rangle=\left\langle n_{i}\right\rangle\left\langle n_{j}\right\rangle$. Other forms of the variances and covariances could be assumed (Bolker and Pacala 1997), but we verify our assertion by noting that the behaviour of the SMEs closely matches the average behaviour of the stochastic system under this choice (see Sect. 3).

Note that we could also arrive at the system of SMEs by consideration of the net flux of cells between compartments (as in Anguige and Schmeiser 2009), noting that

$$
\frac{\mathrm{d}\left\langle\rho_{j}\right\rangle}{\mathrm{d} t}=T_{j-1}^{+}(\boldsymbol{\rho})\left\langle\rho_{j-1}\right\rangle+T_{j+1}^{-}(\boldsymbol{\rho})\left\langle\rho_{j+1}\right\rangle-\left(T_{j}^{+}(\boldsymbol{\rho})+T_{j}^{-}(\boldsymbol{\rho})\right)\left\langle\rho_{j}\right\rangle,
$$

where the vector $\boldsymbol{\rho}=\left(\left\langle\rho_{1}\right\rangle,\left\langle\rho_{2}\right\rangle, \ldots,\left\langle\rho_{k}\right\rangle\right)$, and applying similar moment closure assumptions of the form $\left\langle\rho_{i} \rho_{j}\right\rangle=\left\langle\rho_{i}\right\rangle\left\langle\rho_{j}\right\rangle$. In either case, we obtain

$$
\begin{aligned}
\frac{\mathrm{d}\left\langle\rho_{j}\right\rangle}{\mathrm{d} t}= & \frac{1}{h^{2}}\left(\left\langle\rho_{j+1}\right\rangle\left(1-\alpha\left\langle\rho_{j+2}\right\rangle\right)+\left\langle\rho_{j-1}\right\rangle\left(1-\alpha\left\langle\rho_{j-2}\right\rangle\right)\right. \\
& -\left\langle\rho_{j}\right\rangle\left[\left(1-\left\langle\rho_{j+1}\right\rangle\right)\left(1-\alpha\left\langle\rho_{j-1}\right\rangle\right)+\left\langle\rho_{j+1}\right\rangle\left(1-\alpha\left\langle\rho_{j+2}\right\rangle\right)\right. \\
& \left.\left.+\left\langle\rho_{j-1}\right\rangle\left(1-\alpha\left\langle\rho_{j-2}\right\rangle\right)+\left(1-\left\langle\rho_{j-1}\right\rangle\right)\left(1-\alpha\left\langle\rho_{j+1}\right\rangle\right)\right]\right),
\end{aligned}
$$

for $j=3,4, \ldots, k-2$ (where similar equations can be derived in compartments 1 , $2, k-1$, and $k$ ).

Taking the continuum limit in space (letting the linear dimension of each compartment, $h$, tend to zero) in the SMEs leads us to the PDE of Anguige and Schmeiser (2009) for the evolution of cell density across our domain,

$$
\frac{\partial \rho}{\partial t}=\frac{\partial}{\partial x}\left(D(\rho) \frac{\partial \rho}{\partial x}\right)
$$

with

$$
\frac{\partial \rho}{\partial x}=0
$$

at $x=0$ and $x=1$, and

$$
D(\rho)=3 \alpha\left(\rho-\frac{2}{3}\right)^{2}+1-\frac{4 \alpha}{3} .
$$

Thus, we have a continuous, deterministic population-level model of cell density in the form of a non-linear diffusion equation with diffusivity $D(\rho)$.

\section{Comparison of the Stochastic and Deterministic Models}

We consider the behaviour of our model with initial cell numbers given by

$$
n_{i}(0)=S e^{-2 x_{i}} \sin ^{2}\left(4 \pi x_{i}\right)
$$


where $x_{i}$ is the $x$ co-ordinate of the $i$ th lattice point, so that we can examine the behaviour of our models with varying initial cell densities across the domain. These initial conditions were used by Anguige and Schmeiser (2009).

We can approximate a solution to both the PDE (2) and to the system of SMEs (1) using finite difference methods (Morton and Mayers 2005). We find that, for $\alpha<0.75$, the stochastic system approaches a homogeneous steady state (data not shown). This convergence to steady state in the low $\alpha$ regime occurs independently of the initial conditions that we choose, and independently of the number of compartments on the domain. We also notice that the numerical solution of the PDE and SMEs matches the average behaviour of the stochastic system closely.

For $\alpha>0.75$, however, we find that the diffusivity, $D(\rho)$, becomes negative for certain cell densities. In this $\alpha$ regime, we find that the stochastic system can admit cell clustering. However, as noted by Anguige and Schmeiser (2009), due to the ill-posedness of the PDE we cannot numerically approximate its solution. We can, however, numerically approximate the solution of the SMEs, and we find that this matches the average behaviour of the stochastic system (see Fig. 1). We display the data in our images at the given times for comparison with the paper by Anguige and Schmeiser (2009).

If we increase the total number of compartments (varying $h$ and $S$ appropriately), and reduce the initial number of cells in each compartment by the same factor (so that the density of cells remains the same across the domain), we find that, whereas we might expect the system to display similar behaviour, it instead admits different
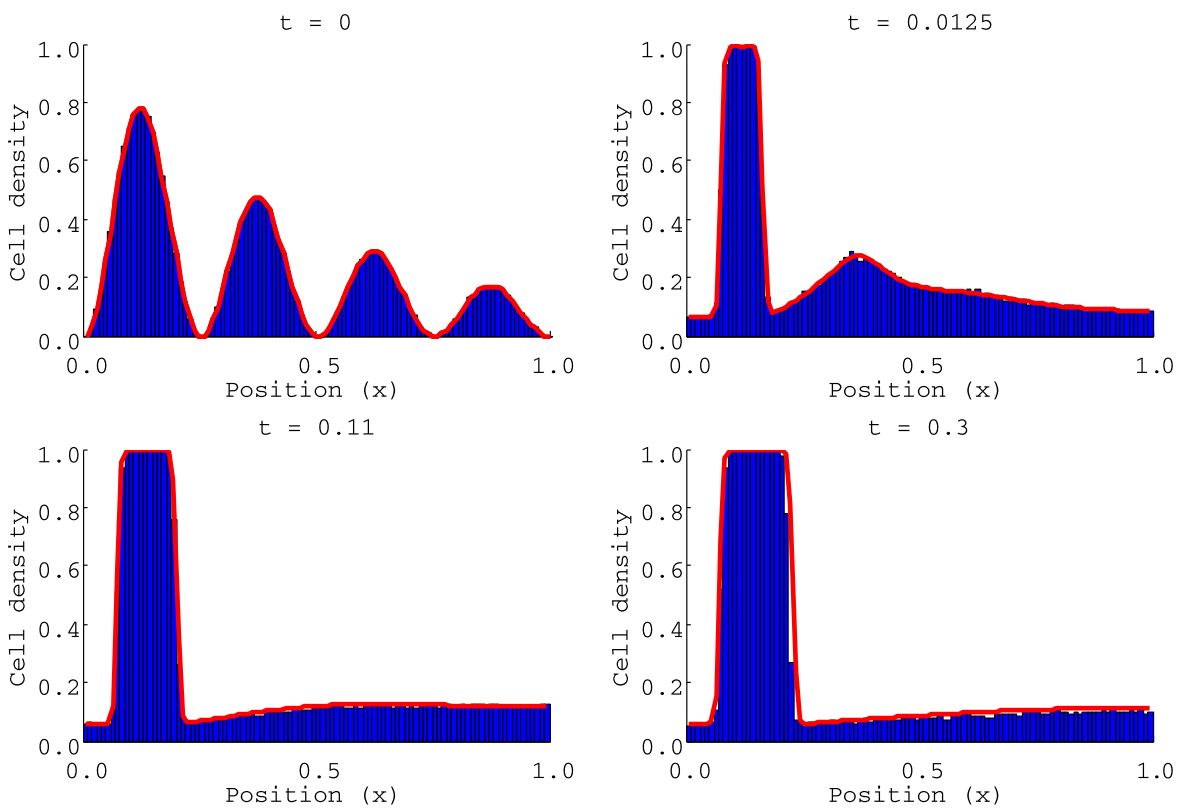

Fig. 1 Average of 100 stochastic simulations (blue) with $S=100$, initial cell density given by (3), and numerical solution of the corresponding SMEs (red), with 100 compartments and $\alpha=0.95$ (Colour figure online) 
types of cell clustering (with the appearance of multiple peaks possible). This lack of convergence appears to be related to the ill-posedness of the PDE: in the low $\alpha$ regime, where the PDE is well-posed, the behaviour of the system is identical for varying numbers of compartments (if the initial cell density is maintained when the number of compartments is varied, as described above). This also suggests that the volume filling aspect of our model (determined by the carrying capacity of an individual compartment, $S$ ) is vital in controlling the type of cell clustering that we see, with the type of clustering dependent on the number of compartments, $k$.

Whilst the results of the deterministic systems are equivalent to the average behaviour of the stochastic system, individual simulations, under certain conditions, can give qualitatively different behaviour. In particular, in the high $\alpha$ regime, starting with homogeneous initial conditions, we find that an individual stochastic simulation can admit cell clustering whereas the deterministic systems remain at this homogeneous steady state. Linear stability analysis of the deterministic systems indicates that the steady state is unstable (Anguige and Schmeiser 2009). This supports our observations, since small perturbations are inherently present in the stochastic simulations (but not in the averaged behaviour).

We note that, after cell clusters are established in the system, the number of peaks stays fixed over very long timescales. For example, for an individual simulation, with an initial homogeneous cell density of 0.6 , we note that there are three peaks in the system at $t=50, t=100$, and $t=200$.

We find that different types of cell clustering can be admitted from the same homogeneous initial conditions. For 100 simulations with $\alpha=0.95,100$ compartments and initial cell density of 0.3 everywhere, we find that at $t=0.2$ we have no peaks in five cases, one peak in 53 cases, two peaks in 41 cases, and three peaks once. For an example of a simulation where two peaks appeared, see Fig. 2.

We also find that varying the magnitude of the initial homogeneous cell density affects the percentage of simulations in which we see cell clustering at some predetermined time later (see Fig. 3). We see that multiple peaks are most likely when we start with a high initial homogeneous density. If the initial cell density is below a critical level, then no cell clustering will occur. If we increase the initial cell density, more peaks are likely. We also notice from Fig. 3 that the number of peaks appearing is variable for the same initial conditions. This further indicates that stochastic effects are important in determining the qualitative behaviour of our system. For an example of a simulation with eight peaks appearing by $t=0.2$, see Fig. 4 .

We notice that when we see cell clustering in the stochastic system and in the behaviour of the stochastic mean equations, the PDE is ill-posed. Further, in our exploration of other systems, when the PDE is not ill-posed we do not see cell clustering. For example, if the transition probabilities are constant, the PDE corresponding to the individual-based model is the diffusion equation, and the system approaches a homogeneous steady state irrespective of the initial conditions. Similar behaviour can be found if the transition probabilities from Sect. 2.1 are changed so that cells adhere to other cells in the same compartment, as we illustrate in the discussion.

Whilst cell clustering is a common feature of biological systems, such behaviour often occurs in systems where multiple cell species interact (Steinberg 1962a, 1962b, 1962c). This leads us to consider the incorporation of two cell species in our model. 

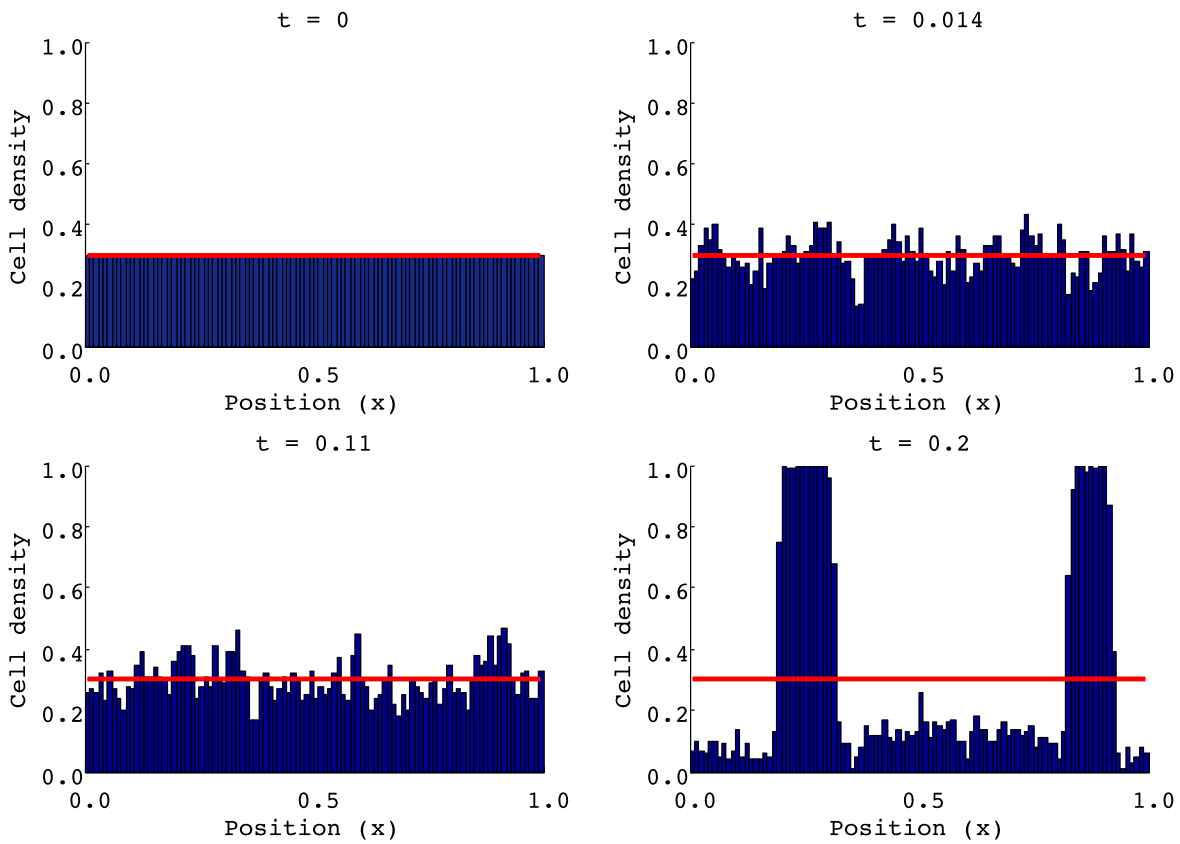

Fig. 2 Individual stochastic simulation (blue) with $S=100$, initial homogeneous cell density of 0.3 , and numerical solution of the corresponding SMEs (red), with 100 compartments and $\alpha=0.95$ (Colour figure online)

\section{Two Cell Species}

We investigate the potential of this model to display behaviour predicted by the differential adhesion hypothesis of Foty and Steinberg (2005) by introducing a second cell species, where the vector $\mathbf{m}=\left(m_{1}, m_{2}, \ldots, m_{k}\right)$ represents the number of cells of the second species in each compartment. We modify the transition rates so that

$$
\begin{aligned}
& T_{i}^{N+}(\mathbf{n}, \mathbf{m})=\frac{1}{h^{2}}\left(1-\alpha_{11} \frac{n_{i-1}}{S}\right)\left(1-\alpha_{12} \frac{m_{i-1}}{S}\right)\left(1-\frac{n_{i+1}+m_{i+1}}{S}\right), \\
& T_{i}^{N-}(\mathbf{n}, \mathbf{m})=\frac{1}{h^{2}}\left(1-\frac{n_{i-1}+m_{i-1}}{S}\right)\left(1-\alpha_{12} \frac{m_{i+1}}{S}\right)\left(1-\alpha_{11} \frac{n_{i+1}}{S}\right), \\
& T_{i}^{M+}(\mathbf{n}, \mathbf{m})=\frac{1}{h^{2}}\left(1-\alpha_{22} \frac{m_{i-1}}{S}\right)\left(1-\alpha_{21} \frac{n_{i-1}}{S}\right)\left(1-\frac{n_{i+1}+m_{i+1}}{S}\right), \\
& T_{i}^{M-}(\mathbf{n}, \mathbf{m})=\frac{1}{h^{2}}\left(1-\frac{n_{i-1}+m_{i-1}}{S}\right)\left(1-\alpha_{21} \frac{n_{i+1}}{S}\right)\left(1-\alpha_{22} \frac{m_{i+1}}{S}\right) .
\end{aligned}
$$

According to these transition rates, cells of each type can move into a neighbouring compartment if that compartment is not full, but at a reduced rate if there are lots of cells of either type in the other neighbouring compartment (with the reduced rate being governed by different adhesion coefficients for each neighbouring cell type). Adhesion coefficients $\alpha_{i j}$ represent the adhesion of species $i$ to species $j$, so that $\alpha_{11}$ 


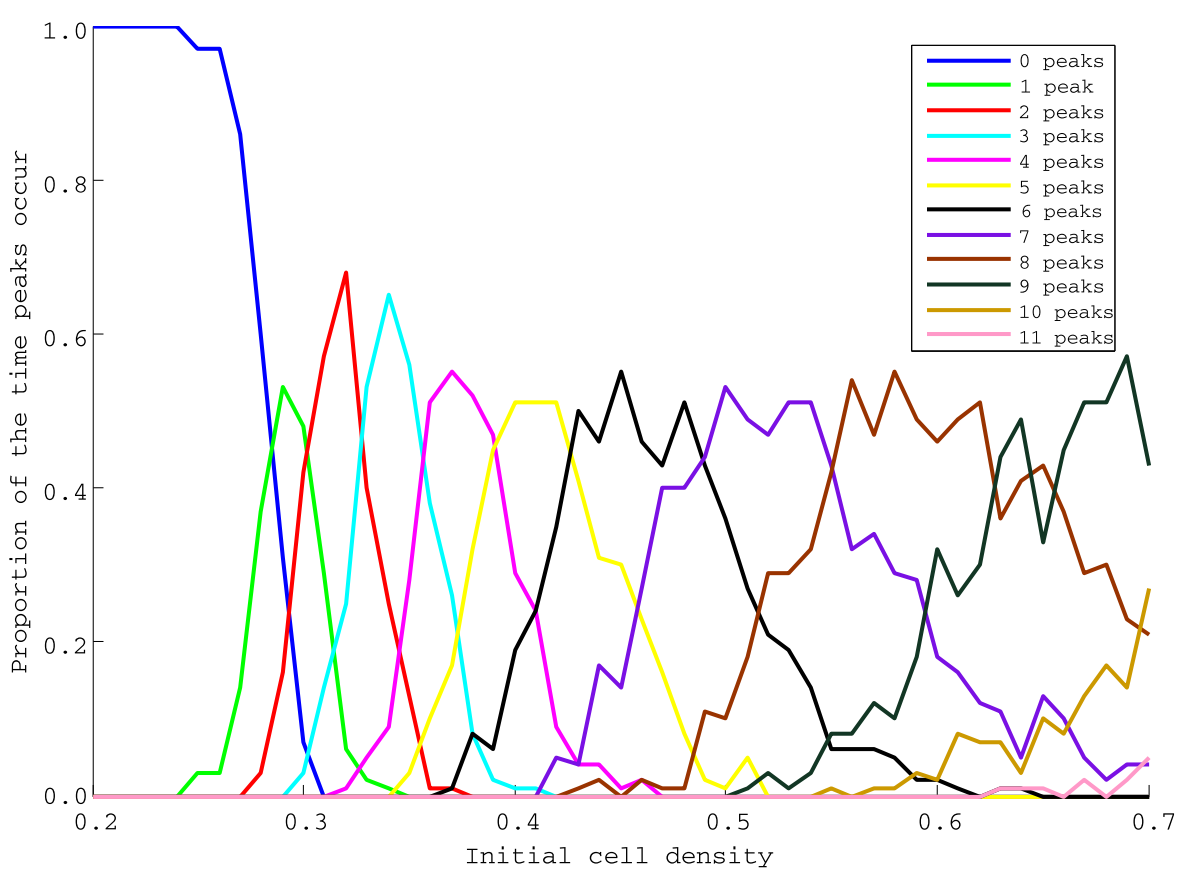

Fig. 3 Proportion of simulations where peaks occur at $t=0.2$ in the stochastic system for homogeneous initial cell density varying from 0.2 to 0.7 , for $\alpha=0.95$. Proportions have been calculated from 100 simulations for each initial cell density (Colour figure online)

represents the adhesion of cell species 1 to itself, with $\alpha_{22}$ representing the adhesion of species 2 to itself. In this work, we shall set $\alpha_{12}$ equal to $\alpha_{21}$, so that the interspecies adhesion coefficients are equal.

Foty and Steinberg (2005) assert that cell species rearrange to form the most thermodynamically stable configuration. In particular, for two cell species, $m$ and $n$, if $w_{m m}, w_{n n}$, and $w_{m n}$ are the work done in the formation of adhesion bonds per unit area between species $m$ and $m, n$ and $n$, and $m$ and $n$, respectively, then different rearrangements will be reached depending on their relative values.

Here, we expect the configuration reached to be dependent on the adhesion coefficients in a similar fashion. Whilst we might expect slightly different behaviour from that observed experimentally by Foty and Steinberg (2005) because of the onedimensional nature of the model and also the volume filling aspect (with multiple cells clustering in each compartment, rather than lots of cells side-by-side), we find that various rearrangements are possible dependent on the adhesion parameters.

Armstrong et al. (2006) showed that their continuous model was capable of replicating experimental cell sorting, ending in various possible configurations, including complete sorting, engulfment, and mixing. We find that these three configurations are also possible in our system, and example simulations are shown in Fig. 5. The type of clustering established by $t=5$ did not change by $t=100$ (data not shown).

We find that if the inter-species adhesion parameters are large, or the intra-species adhesion parameters are small, then mixing occurs. The engulfment of species 1 by 

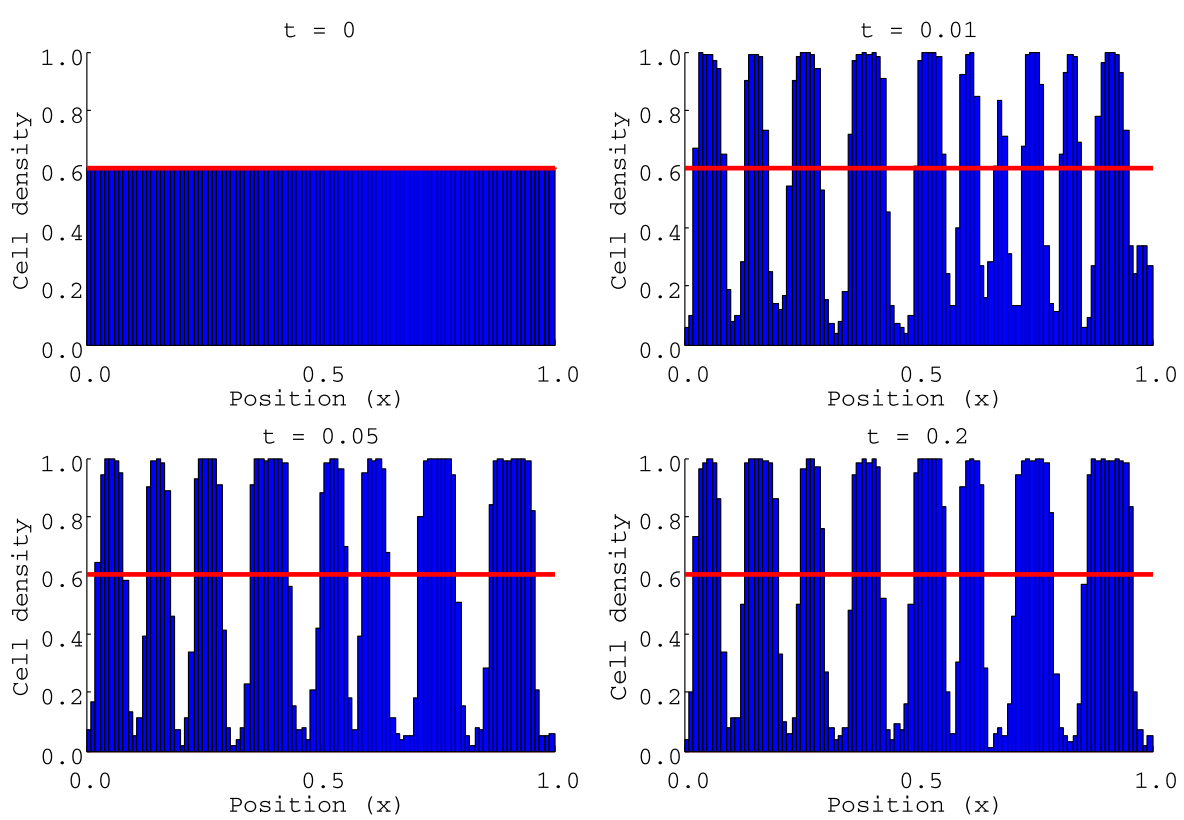

Fig. 4 Individual stochastic simulation (blue) with $S=100$, initial homogeneous cell density of 0.6 , and numerical solution of the corresponding SMEs (red), with 100 compartments and $\alpha=0.95$ (Colour figure online)

species 2 occurs if the inter-species adhesion parameters are small, with $\alpha_{11}$ large and $\alpha_{22}$ small (and vice versa for the engulfment of species 2 by species 1 ). Complete sorting occurs if either both inter-species adhesion coefficients are small and both intra-species adhesion coefficients are large, or if the inter-species adhesion coefficients are zero and at least one of the intra-species adhesion coefficients is large.

During morphogenesis, embryos change size, with cell migration taking place on top of this in order to form components of the body. Consequently, whilst the incorporation of two cell species is important, an equally important consideration when modelling biological development is domain growth.

\section{Domain Growth}

In order to incorporate domain growth into our model, we include a growth event as an alternative to cell movement in the Gillespie stochastic simulation algorithm. In some earlier models, such as that of Baker et al. (2010), a growth event involves the splitting of a compartment (chosen at random) into two new compartments (both of the same linear dimension as the original compartment), with the cells in the original compartment divided (according to some distribution) between the two new compartments. However, a compartment split then instantaneously reduces the density of cells at that point on the domain drastically, which seems an unlikely consequence of growth in a biological system. In a model that admits cell clustering, this type of 

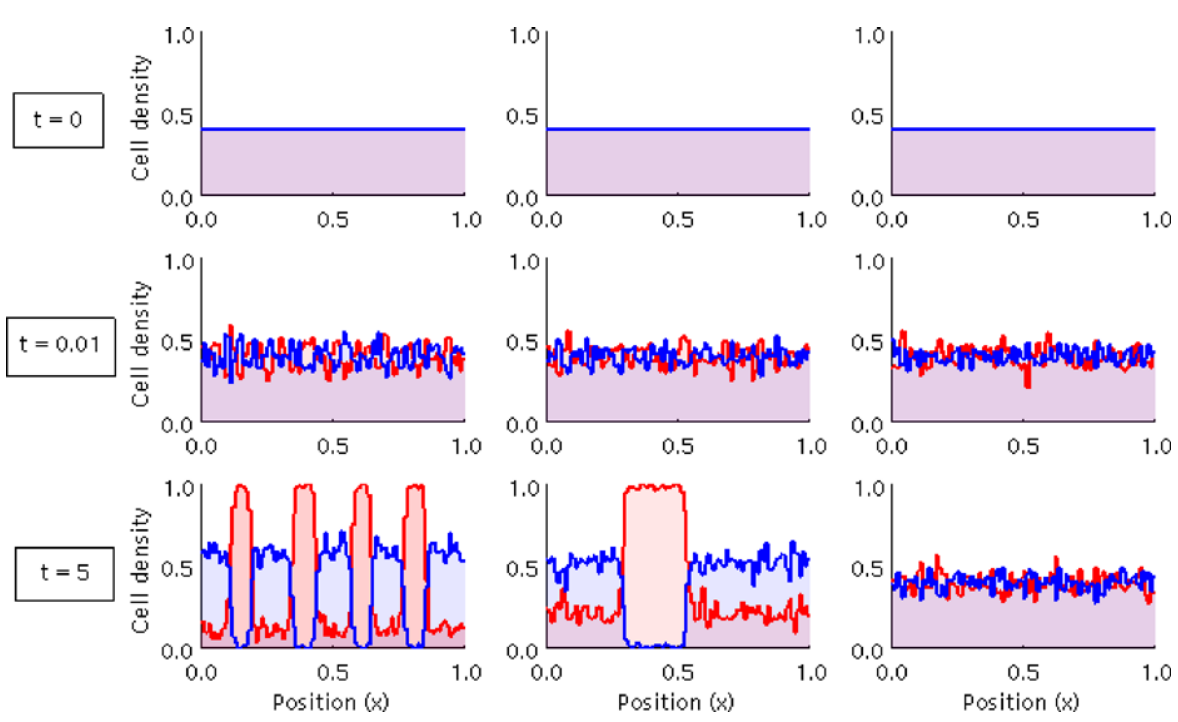

Fig. 5 Individual stochastic simulations of the two species model with transition probabilities given by equations (4), with $S=100$, and initial homogeneous cell density 0.4 of both type 1 (blue) and type 2 (red). Left: Complete sorting; $\alpha_{11}=0.1, \alpha_{22}=0.9$ and $\alpha_{12}=\alpha_{21}=0.0$. Middle: Engulfment; $\alpha_{11}=0.1$, $\alpha_{22}=0.9$ and $\alpha_{12}=\alpha_{21}=0.2$. Right: Mixing; $\alpha_{11}=0.1, \alpha_{22}=0.9$ and $\alpha_{12}=\alpha_{21}=0.6$ (Colour figure online)

growth can lead to unrealistic features such as instantaneous peak splitting. We therefore present a novel approach. Whilst we only consider the case of an exponentially growing domain, where each compartment grows at average rate $r S$ (where $S$ is the current carrying capacity of the compartment concerned), the method can be adapted for systems where compartment growth rates are dependent on the density of cells in each compartment, which might be more biologically realistic (Lieberman and Glaser 1981).

In our approach, a growth event involves increasing the linear dimension of a compartment (chosen at random) by the extent of a single cell (increasing the carrying capacity of the compartment by one). A growth event in this way only decreases the density of cells in the compartment concerned slightly. When the compartments become large, the distance that cells have to jump between lattice points is also large, which is unrealistic. Therefore, when a compartment carrying capacity reaches a predefined threshold, the compartment splits into two new compartments, each with carrying capacity half of this threshold (thus ensuring that the cell density at any point on our domain does not change by a large amount during any growth event, since, for example, a compartment containing 80 cells and carrying capacity 200 will split into two compartments that contain 40 cells and have carrying capacity 100 , so that cell density is conserved at 0.4 ).

Hence, if we start with $k_{0}$ compartments each with initial carrying capacity $C_{0}$, at any growth event we choose a compartment (at random, with the probability of choosing a certain compartment proportional to its current carrying capacity) to increase in carrying capacity by one cell. When any compartment reaches carrying capacity $2 C_{0}$, say, then it splits into two compartments, each with carrying capacity $C_{0}$, and 

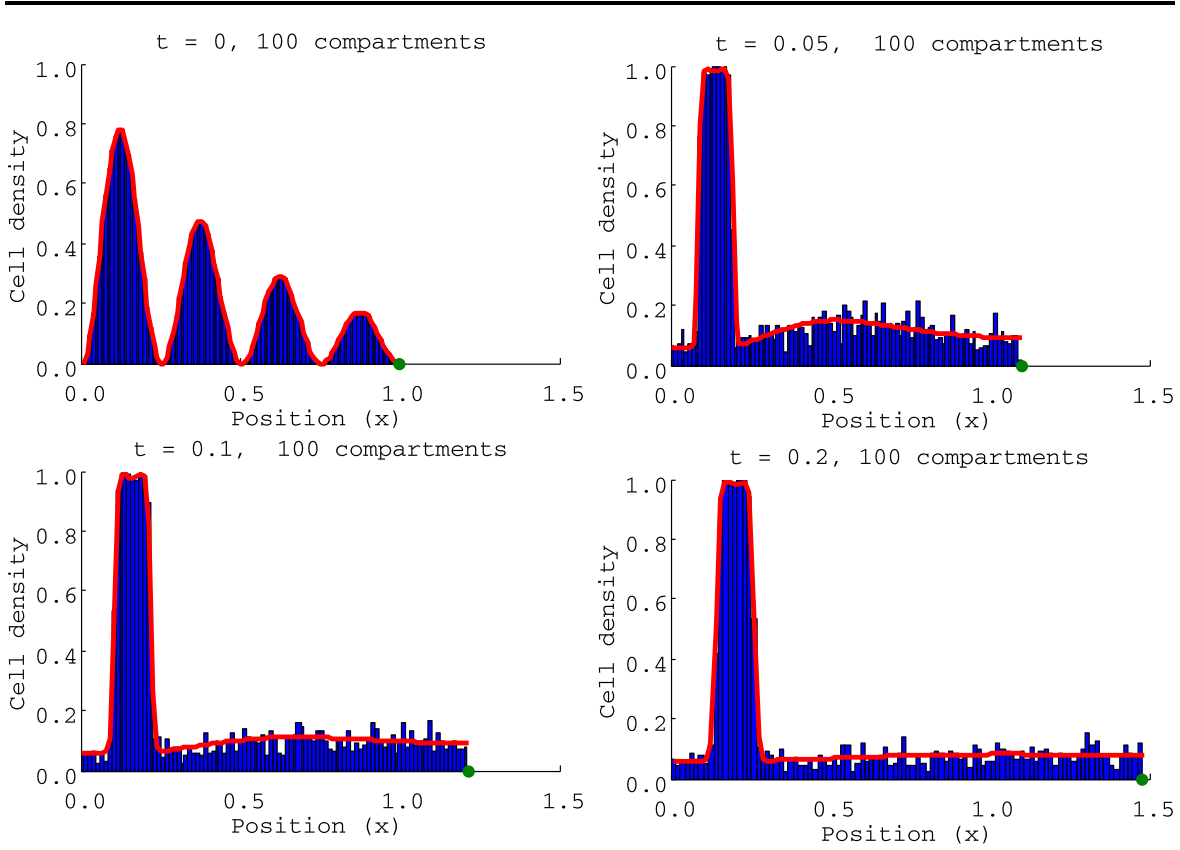

Fig. 6 Individual stochastic simulation with domain growth incorporated, with 100 compartments (each with initial carrying capacity $C_{0}=100$ ) initially, $\alpha=0.95$, growth rate $r=2$ and initial distribution of cells given by (3). The red line is the solution of the corresponding SMEs, and the green dot indicates the size of the domain of the stochastic system. None of the compartments reach the critical carrying capacity of 200 (at which the compartment concerned would be split into two compartments) by $t=0.2$; consequently, there are still 100 compartments in each of the displayed figures (Colour figure online)

the cells in the original compartment are split evenly between the new compartments (if there are an odd number of cells in the parent compartment, then one of the new compartments, chosen uniformly at random, will contain an extra cell). We assume that the size of each compartment is proportional to its carrying capacity (so that the domain size can increase whilst the number of compartments stays constant, as in Fig. 6).

We must modify the transition rates in Sect. 2.1, in order to consider a domain with unequal compartment sizes (since, if the distance between lattice points is larger, we expect the probability of a cell jumping between the lattice points to be reduced). Motivated by the discretisation of a PDE on a non-uniform domain (see the Appendix), we use transition rates

$$
\begin{aligned}
& T_{i}^{+}(\mathbf{n})=\frac{1}{h_{i+1} h_{i+\frac{1}{2}}}\left(1-\alpha \frac{n_{i-1}}{S_{i-1}}\right)\left(1-\frac{n_{i+1}}{S_{i+1}}\right), \\
& T_{i}^{-}(\mathbf{n})=\frac{1}{h_{i} h_{i+\frac{1}{2}}}\left(1-\frac{n_{i-1}}{S_{i-1}}\right)\left(1-\alpha \frac{n_{i+1}}{S_{i+1}}\right),
\end{aligned}
$$

for $i=2,3, \ldots, k-1$ (with similar expressions for $i=1$ and $i=k$ ) where $S_{i}(t)$ is the carrying capacity of compartment $i$ at time $t$ and $h_{i}$ is defined as in Sect. 2.1 (with 

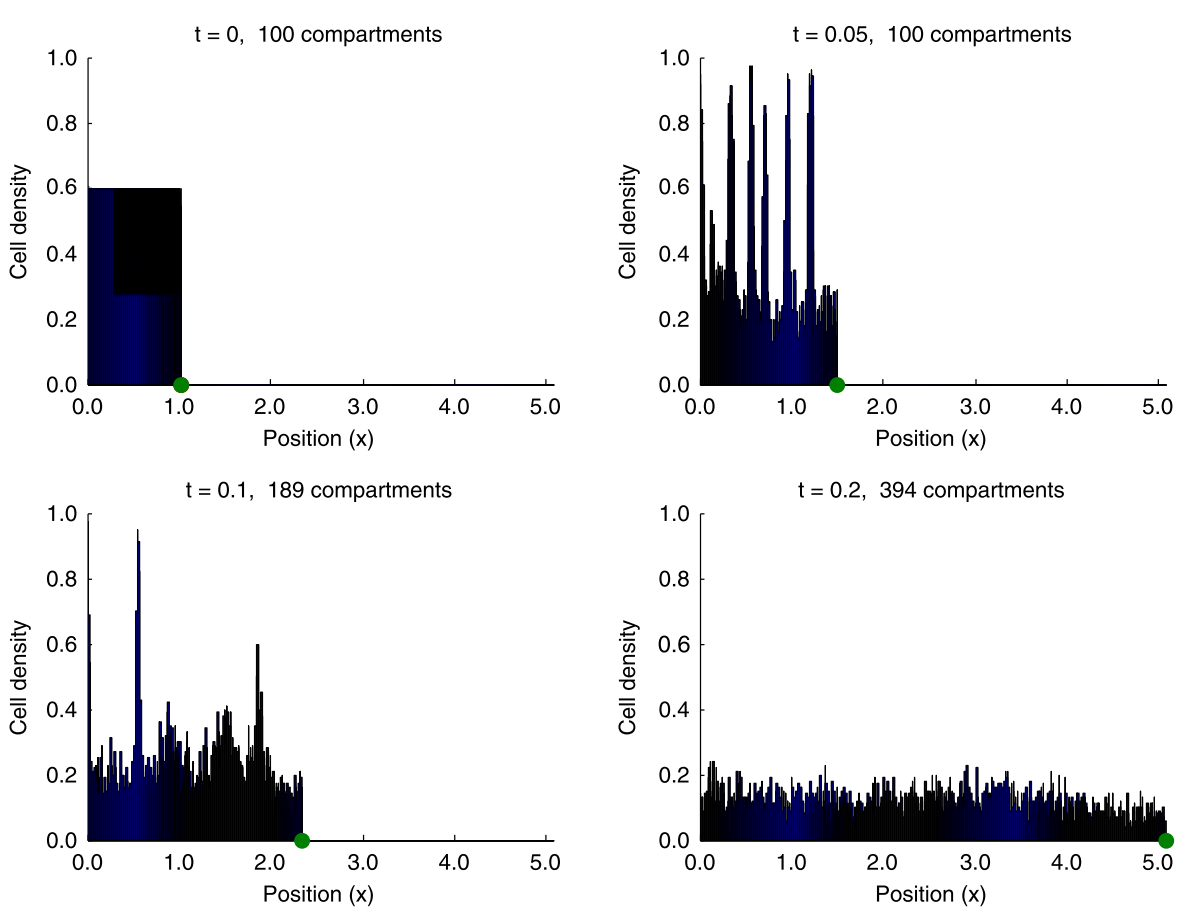

Fig. 7 Individual stochastic simulation with domain growth incorporated with 100 compartments (each with initial carrying capacity $C_{0}=100$ ) initially, $\alpha=0.8$, growth rate $r=8$ and initial homogeneous cell density equal to 0.6 . The green dot indicates the size of the domain (Colour figure online)

$h_{i+\frac{1}{2}}$ defined to be the mean of $h_{i}$ and $h_{i+1}$ ). We note that the linear dimension of a single cell is $1 / k_{0} C_{0}$, so that

$$
h_{i}=\frac{\frac{1}{2}\left(S_{i-1}+S_{i}\right)}{k_{0} C_{0}},
$$

for $i=2,3, \ldots, k$ (with similar expressions for $i=1$ and $i=k+1$ ).

We can also derive SMEs for both $\left\langle S_{i}\right\rangle(t)$ and $\left\langle n_{i}\right\rangle(t)$ using a master equation formulation approach as in the stationary domain case (Baker et al. 2010). The SMEs are valid up to the time of the first compartment split (see Fig. 6).

We find that, in the low $\alpha$ regime, the system approaches a state with cells equally distributed across the domain, but in the high $\alpha$ regime, cell clustering can occur, as in the stationary domain case. Numerical simulations indicate that the probability of peaks appearing is decreased by increasing rate of domain growth. We observe that, over short timescales, for small growth rates cell clusters can still form, but for large growth rates they cannot. For intermediate growth rates, cell clusters form initially but are destroyed eventually (since the domain grows exponentially), as shown in Fig. 7. 


\section{Discussion}

Cell migration and adhesion are important in various areas of biology, and especially important during development. Mathematical modelling provides a framework where hypotheses can be tested and refined, and experimentally verifiable predictions can be made.

We have considered a stochastic model of cell migration that incorporates both cell-cell adhesion and volume filling, and also two corresponding deterministic systems in the form of a set of SMEs and a non-linear diffusion equation. We find that, for low values of the adhesion parameter, $\alpha$, numerical solutions of the deterministic systems match the behaviour of the stochastic system closely, and a homogeneous steady state is reached. For large values of $\alpha$, on the other hand, the PDE becomes illposed, and gives nonsensical solutions for cell densities where the diffusivity, $D(\rho)$, becomes negative. This means that we are unable to model biological systems where cell clustering occurs using this PDE model. The numerical solution of the SMEs, however, matches the average behaviour of the stochastic system, and various types of cell clustering can occur (for example, different numbers of peaks).

We note, however, that whilst the SMEs depict the average behaviour of the stochastic system, individual stochastic simulations can vary dramatically from the behaviour predicted by the SMEs. In particular, cell clustering could occur in one realisation of the stochastic system when it does not in the SMEs. We also noticed that different types of cell clustering may occur from the same initial conditions in the stochastic system.

It is possible to consider a system in which cells adhere to other cells in the same compartment, so that the transition probabilities are given by

$$
\begin{aligned}
& T_{i}^{+}(\mathbf{n})=\frac{d}{h^{2}}\left(1-\alpha \frac{n_{i}}{S}\right)\left(1-\frac{n_{i+1}}{S}\right), \\
& T_{i}^{-}(\mathbf{n})=\frac{d}{h^{2}}\left(1-\frac{n_{i-1}}{S}\right)\left(1-\alpha \frac{n_{i}}{S}\right) .
\end{aligned}
$$

This system leads to a similar PDE, but with diffusivity

$$
D(\rho)=\alpha \rho^{2}-2 \alpha \rho+1 .
$$

In this case, the diffusivity does not become negative for any cell densities with $\alpha \in$ $[0,1]$, and the solutions of the system of SMEs and the PDE, as well as simulations of the stochastic model, show that the system tends to the homogeneous steady state. Consequently, this model cannot explain the cell clustering observed in biological systems, and we instead considered the transition probabilities of Sect. 2.1, initially considered by Anguige and Schmeiser (2009).

Recognising that cell-cell adhesion often occurs in systems where multiple cell species interact, we then incorporated a second cell species into our stochastic system, and found that various qualitatively-different behaviours were possible (such as the engulfment of one species by another, complete cell sorting, and the retention of random mixing long-term) depending on the different adhesion parameters (both 
intra- and inter-species). This is consistent with the behaviour observed by Armstrong et al. (2006).

Whilst the incorporation of multiple cell species into our model is important, it is also important to consider domain growth when modelling biological development. A new method for incorporating domain growth via the incremental increase of carrying capacities of individual compartments was proposed here. SMEs can again be derived, and they match the behaviour of the stochastic system short-term. We find that domain growth decreases the chance of cell clustering occurring, with the peaks that do form being stretched and shortened by the growth. Peak splitting, which is an artefact of some other stochastic growth simulation techniques if used with models that admit cell clustering (Baker et al. 2010), does not occur.

This work suggests areas for further investigation, so that we can fully understand the mechanisms underlying cell migration and adhesion, particularly during biological development. A sensible next step might be the extension of our model to two or three spatial dimensions, or the incorporation of more cell species. Further theoretical work might then include considering different transition probabilities: a broad framework for this has been developed by Penington et al. (2011).

Acknowledgements RNT would like to thank the Centre for Mathematical Biology, University of Oxford, for the opportunity to carry out this research, the Nuffield Foundation for the bursary that allowed this research to begin, and BBSRC for research funding via the Genes to Organisms doctoral training award. He would also like to thank Endre Suli, Michael Thompson and Nik Cunniffe for helpful discussions and support. CAY would like to thank Christ Church College, Oxford, for a Junior Research Fellowship.

\section{Appendix: Transition Probabilities on a Non-uniform Domain}

When the domain has unequal compartment sizes, the distance that cells jump between neighbouring compartments varies. If this distance is large, then the transition rate between the compartments concerned should be reduced.

We note that, if we consider a stochastic system with transition probabilities of the form

$$
T_{i}^{ \pm}=D / h^{2},
$$

where diffusivity $D$ is constant, we can derive the PDE

$$
\frac{\partial \rho}{\partial t}=-\frac{\partial}{\partial x}\left(-D \frac{\partial \rho}{\partial x}\right),
$$

as discussed by Baker et al. (2010).

If we consider approximating a solution to this PDE on a non-uniform domain (Morton and Mayers 2005), we obtain

$$
\begin{aligned}
\frac{n_{i}^{k+1}-n_{i}^{k}}{\Delta t}= & \frac{2 D}{h_{i+1}\left(h_{i+1}+h_{i+2}\right)} n_{i+1}+\left(\frac{-2 D}{h_{i}\left(h_{i}+h_{i+1}\right)}+\frac{-2 D}{h_{i+1}\left(h_{i}+h_{i+1}\right)}\right) n_{i} \\
& +\frac{2 D}{h_{i}\left(h_{i-1}+h_{i}\right)} n_{i-1},
\end{aligned}
$$


where the $h_{i}$ s are defined as in Sect. 2.1. Assuming that the number of cells evolves according to

$$
\frac{\partial n_{i}}{\partial t}=T_{i+1}^{-} n_{i+1}+T_{i-1}^{+} n_{i-1}-\left(T_{i}^{-}+T_{i}^{+}\right) n_{i},
$$

we deduce that

$$
T_{i}^{+}=\frac{2 D}{h_{i+1}\left(h_{i}+h_{i+1}\right)},
$$

with a similar expression obtainable for $T_{i}^{-}$. For ease of notation, we define

$$
h_{i+\frac{1}{2}}=\frac{1}{2}\left(h_{i}+h_{i+1}\right) \text {. }
$$

The above consideration motivates the pre-factor in our transition rates on a nonuniform domain, for $i=2,3, \ldots, k-1$ (where the cell density in compartment $i$ is $\left.n_{i} / S_{i}\right)$, given by

$$
\begin{aligned}
& T_{i}^{+}(\mathbf{n})=\frac{d}{h_{i+1} h_{i+\frac{1}{2}}}\left(1-\alpha \frac{n_{i-1}}{S_{i-1}}\right)\left(1-\frac{n_{i+1}}{S_{i+1}}\right), \\
& T_{i}^{-}(\mathbf{n})=\frac{d}{h_{i} h_{i+\frac{1}{2}}}\left(1-\frac{n_{i-1}}{S_{i-1}}\right)\left(1-\alpha \frac{n_{i+1}}{S_{i+1}}\right),
\end{aligned}
$$

with similar expressions derived for $i=1$ and $i=k$. Strictly speaking, we have assumed that the compartment edges are halfway between the lattice points.

\section{References}

Alberts, B., Bray, D., Lewis, J., Raff, M., Roberts, K., \& Watson, J. D. (1994). Molecular biology of the cell (3rd ed.). New York: Garland.

Anguige, K. (2011). A one-dimensional model for the interaction between cell-to-cell adhesion and chemotactic signalling. Eur. J. Appl. Math., 22(4), 291-316.

Anguige, K., \& Schmeiser, C. (2009). A one-dimensional model of cell diffusion and aggregation, incorporating volume filling and cell-to-cell adhesion. J. Math. Biol., 58(3), 395-427.

Armstrong, N. J., Painter, K. J., \& Sherratt, J. A. (2006). A continuum approach to modelling cell-cell adhesion. J. Theor. Biol., 243(1), 98-113.

Baker, R. E., Yates, C. A., \& Erban, R. (2010). From microscopic to macroscopic descriptions of cell migration on growing domains. Bull. Math. Biol., 72(3), 719-762.

Berg, H. C. (1975). How bacteria swim. Sci. Am., 233(2), 36-44.

Berg, H. C. (1993). Random walks in biology. Princeton: Princeton University Press.

Bolker, B., \& Pacala, S. W. (1997). Using moment equations to understand stochastically driven spatial pattern formation in ecological systems. Theor. Popul. Biol., 52(3), 179-197.

Brenner, M. P., Levitov, L. S., \& Budrene, E. O. (1998). Physical mechanisms for chemotactic pattern formation by bacteria. Biophys. J., 74(4), 1677-1693.

Crampin, E. J., Gaffney, E. A., \& Maini, P. K. (1999). Reaction and diffusion on growing domains: scenarios for robust pattern formation. Bull. Math. Biol., 61(6), 1093-1120.

Erban, R., \& Othmer, H. G. (2004). From individual to collective behavior in bacterial chemotaxis. SIAM J. Appl. Math., 65(2), 361-391.

Foty, R. A., \& Steinberg, M. S. (2005). The differential adhesion hypothesis: a direct evaluation. Dev. Biol., 278(1), 255-263. 
Gillespie, D. T. (1977). Exact stochastic simulation of coupled chemical reactions. J. Phys. Chem., 81(1), 2340-2361.

Keller, E. F., \& Segel, L. A. (1970). Initiation of slime mold aggregation viewed as an instability. J. Theor. Biol., 26(3), 399-415.

Keller, E. F., \& Segel, L. A. (1971a). Model for chemotaxis. J. Theor. Biol., 30(2), 225-234.

Keller, E. F., \& Segel, L. A. (1971b). Traveling bands of chemotactic bacteria: a theoretical analysis. J. Theor. Biol., 30(2), 235-248.

Khain, E., Katakowski, M., Hopkins, S., Szalad, A., Zheng, X., Jiang, F., \& Chopp, M. (2011). Collective behavior of brain tumor cells: the role of hypoxia. Phys. Rev. E, 83(3), 031920.

Landman, K. A., Pettet, G. J., \& Newgreen, D. F. (2003). Mathematical models of cell colonization of uniformly growing domains. Bull. Math. Biol., 65(2), 235-262.

Lieberman, M. A., \& Glaser, L. (1981). Density dependent regulation of cell growth: an example of a cell-cell recognition phenomenon. J. Membr. Biol., 11, 1-11.

Maini, P. K., \& Solursh, M. (1991). Cellular mechanisms of pattern formation in the developing limb. Int. Rev. Cytol., 129, 91-133.

Mooney, J. R., \& Nagorcka, B. N. (1985). Spatial patterns produced by a reaction-diffusion system in primary hair follicles. J. Theor. Biol., 115(2), 299-317.

Morton, K. W., \& Mayers, D. F. (2005). Numerical solution of partial differential equations: an introduction. Cambridge: Cambridge University Press.

Murray, J. D. (2002). Mathematical biology (3rd ed.). Berlin: Springer.

Othmer, H. G., \& Hillen, T. (2011). The diffusion limit of transport equations II: chemotaxis equations. SIAM J. Appl. Math., 62(4), 1222-1250.

Othmer, H. G., \& Schaap, P. (1998). Oscillatory cAMP signaling in the development of Dictyostelium discoideum. Comments Theor. Biol., 5, 175-282.

Othmer, H. G., \& Stevens, A. (1997). Aggregation, blowup, and collapse: the ABC's of taxis in reinforced random walks. SIAM J. Appl. Math., 57(4), 1044-1081.

Othmer, H. G., Dunbar, S. R., \& Alt, W. (1988). Models of dispersal in biological systems. J. Math. Biol., 26, 263-298.

Palsson, E., \& Othmer, H. G. (2000). A model for individual and collective cell movement in Dictyostelium discoideum. Proc. Natl. Acad. Sci. USA, 97(19), 10448-10453.

Patlak, C. S. (1953). Random walk with persistence and external bias. Bull. Math. Biophys., 15(3), 311338.

Penington, C. J., Hughes, B. D., \& Landman, K. A. (2011). Building macroscale models from microscale probabilistic models: a general probabilistic approach for nonlinear diffusion and multispecies phenomena. Phys. Rev. E, 84(4), 041120.

Simpson, M. J., Landman, K. A., Hughes, B. D., \& Fernando, A. E. (2010a). A model for mesoscale patterns in motile populations. Physica A, 389(7), 1412-1424.

Simpson, M. J., Towne, C., McElwain, D. L. S., \& Upton, Z. (2010b). Migration of breast cancer cells: understanding the roles of volume exclusion and cell-to-cell adhesion. Phys. Rev. E, 82(4), 041901.

Steinberg, M. S. (1962a). On the mechanism of tissue reconstruction by dissociated cells, I. Population kinetics, differential adhesiveness, and the absence of directed migration. Proc. Natl. Acad. Sci. USA, 48(9), 1577-1582.

Steinberg, M. S. (1962b). Mechanism of tissue reconstruction by dissociated cells, II. Time-course of events. Science, 137(3532), 762-763.

Steinberg, M. S. (1962c). On the mechanism of tissue reconstruction by dissociated cells, III. Free energy relations and the reorganization of fused, heteronomic tissue fragments. Proc. Natl. Acad. Sci. USA, 48(10), 1769-1776.

Woolley, T., Baker, R. E., Gaffney, E. A., \& Maini, P. K. (2011). Influence of stochastic domain growth on pattern nucleation for diffusive systems with internal noise. Phys. Rev. E, 84(4), 041905. 